\title{
An Efficient Class of Exponential Chain Ratio Type Estimator for Finite Population Mean in Double Sampling
}

\author{
Diganta Kalita
}

Department of Statistics, North Lakhimpur College (Autonomous), North Lakhimpur, Assam, India

\section{Email address:}

dkalita.nl@gmail.com

\section{To cite this article:}

Diganta Kalita. An Efficient Class of Exponential Chain Ratio Type Estimator for Finite Population Mean in Double Sampling. Science Journal of Applied Mathematics and Statistics. Vol. 3, No. 6, 2015, pp. 281-287. doi: 10.11648/j.sjams.20150306.17

\begin{abstract}
This paper presents a class of exponential chain ratio type estimator in double sampling for estimating finite population mean of the study variable, when the information on another additional auxiliary variable is known along with the main auxiliary variable. The property of proposed class of estimator has been studied. Comparison has been made with other competitive estimators. The proposed estimator is found to be more efficient both theoretically and empirically.
\end{abstract}

Keywords: Auxiliary Character, Chaining in Double Sampling, Exponential Chain Ratio Type Estimator, Study Variable, MSE and Efficiency

\section{Introduction}

It is well established fact that the uses of auxiliary information in sample survey increases the precision of the estimate of population mean of study variable. W. G. Cochran [5] used auxiliary information and proposed the classical ratio estimator of population mean or total of the study variable $y$. Ratio method of estimation is quite effective when there is a high positive correlation between study and auxiliary variables. In the ratio method of estimation, it is assumed that the auxiliary information is known in advance. However, there are some situations of practical importance where the population mean of auxiliary information $X$ is not known before start of the surveys. In such a situation, a sample of size $n_{1}$ is selected initially by using a suitable sampling design and population mean $\bar{X}$ is estimated, then a sample of size $n\left(n<n_{1}\right)$ is selected to estimate the population mean of the study and auxiliary variables. The second phase sample can either be a subsample of the first phase sample or it can be directly drawn from the given population. This technique is known as double or two phase sampling and was first introduced by Neyman [7].

However, if the population mean $\bar{Z}$ of another auxiliary variable $Z$, closely related to $x$ but compared to $x$ remotely related to $y$ is known (i.e. $\rho_{y x}>\rho_{y z}$ ), it is preferable to estimate $\bar{X}$ by $\bar{X}=\bar{x}_{1} \bar{Z} / \bar{z}_{1}$, which would provide better estimate of $\bar{X}$ than $\bar{x}_{1}$ to the terms of order $o\left(n^{-1}\right)$ if $\rho_{x z} C_{x} / C_{z}>1 / 2$, where $C_{x}, C_{z}$ and $\rho_{y x}, \rho_{y z}, \rho_{x z}$ are coefficient of variation of $x, z$ and correlation coefficient between $y$ and $x ; y$ and $z ; x$ and $z$ respectively. This technique is known as chaining. The chain regression estimator was first introduced by Swain [18].Chand [4], Sukhatme and Chand [16], Kiregyera [9] proposed some chain ratio type estimators based on two auxiliary variates. Srivastava and Jhajj [14], Al-Jararha and Ahmed [1], Pradhan[11], Amin and Hanif [2], Singh and Choudhury [12], Kalita et. al [8] and many authors have suggested some improved chain ratio type estimators using single (or more) auxiliary variable(s) in order to provide better efficiency.

Let us consider a finite population $U=\left(U_{1}, U_{2}, \ldots U_{N}\right)$ of size $\mathrm{N}$ units and the value of the variables on the $i^{\text {th }}$ unit be $\left(y_{i}, x_{i}\right)$, where $i=1,2, \ldots, N$. Let $\bar{Y}=\sum_{i=1}^{N} \frac{y_{i}}{N}$ and $\bar{X}=\sum_{i=1}^{N} \frac{x_{i}}{N}$ be the population means of the study variable $y$ and the auxiliary variable $x$ respectively. For estimating the population mean $\bar{Y}$ of $y$, a simple random sample of size $n$ is drawn without replacement from the population $U$. Then the classical ratio estimator is defined by 


$$
\hat{\bar{Y}}_{R}=\bar{y} \frac{\bar{X}}{\bar{x}}, \text { if } \bar{x} \neq 0
$$

where $\bar{y}$ and $\bar{x}$ are the sample means of $y$ and $x$ respectively based on a sample of size $n$ out of the population of size $N$ units and $\bar{X}$ is the known as population mean of $x$. With known population mean $\bar{X}$, Bahl \& Tuteja [3] suggested the exponential ratio type estimator as

$\hat{\bar{Y}}_{R e}=\bar{y} \exp \left(\frac{\bar{X}-\bar{x}}{\bar{X}+\bar{x}}\right)$ for estimating the population mean $\bar{Y}$.

If the population mean $\bar{X}$ of the auxiliary variable $x$ is not known before start of the survey, a first-phase sample of size $n_{1}$ is drawn from the population, on which only the auxiliary variable $x$ is observed. Then a second phase sample of size $n$ is drawn, on which both study variable $y$ and auxiliary variable $x$ are observed. Let $\bar{x}_{1}=\sum_{i=1}^{n_{1}} \frac{x_{i}}{n_{1}}$ denotes the sample mean of size $n_{1}$ based on the first phase sample and $\bar{y}=\sum_{i=1}^{n} \frac{y_{i}}{n}$ and $\bar{x}=\sum_{i=1}^{n} \frac{x_{i}}{n}$ denote the sample means of variables $Y$ and $X$ respectively, obtained from the second phase sample of size $n$. The ratio estimator in double sampling Sukhatme [17] for estimating population mean $\bar{Y}$ is given by $\bar{y}_{R}^{d}=\left(\frac{\bar{y}}{\bar{x}}\right) \bar{x}_{1}$.

Singh \&Vishwakarma [13] suggested the exponential ratio type estimators for $\bar{Y}$ in double sampling as

$$
\hat{\bar{Y}}_{R e}^{d}=\bar{y} \exp \left(\frac{\bar{x}_{1}-\bar{x}}{\bar{x}_{1}+\bar{x}}\right) \text {.Chand [4] developed a chain ratio }
$$

estimator for the population mean in double sampling as,

\section{Bias and MSE of Case I}

In case I, let us write

$$
e_{0}=(\bar{y}-\bar{Y}) / \bar{Y}, e_{1}=(\bar{x}-\bar{X}) / \bar{X}, e_{1}^{\prime}=\left(\bar{x}_{1}-\bar{X}\right) / \bar{X} \text { and } e_{2}=\left(\bar{z}_{1}-\bar{Z}\right) / \bar{Z} \text { such that }
$$

$$
\left.\begin{array}{l}
E\left(e_{0}\right)=E\left(e_{1}\right)=E\left(e_{1}^{\prime}\right)=E\left(e_{2}\right)=0, \quad E\left(e_{0}^{2}\right)=\frac{1-f}{n} C_{y}^{2}, \quad E\left(e_{1}^{2}\right)=\frac{1-f}{n} C_{x}^{2}, \\
E\left(e_{1}^{\prime 2}\right)=\frac{1-f_{1}}{n_{1}} C_{x}^{2}, \quad E\left(e_{2}^{2}\right)=\frac{1-f_{1}}{n_{1}} C_{z}^{2}, \quad E\left(e_{0} e_{1}\right)=\frac{1-f}{n} C_{y x} C_{x}^{2}, \quad E\left(e_{0} e_{1}^{\prime}\right)=\frac{1-f_{1}}{n_{1}} C_{y x} C_{x}^{2}, \\
E\left(e_{0} e_{2}\right)=\frac{1-f_{1}}{n_{1}} C_{y z} C_{z}^{2}, E\left(e_{1} e_{1}^{\prime}\right)=\frac{1-f_{1}}{n_{1}} C_{x}^{2}, E\left(e_{1} e_{2}\right)=E\left(e_{1}^{\prime} e_{2}\right)=\frac{1-f_{1}}{n_{1}} C_{x z} C_{z}^{2}
\end{array}\right\}
$$

where $f=\frac{n}{N}, f_{1}=\frac{n_{1}}{N}, C_{y}=\frac{S_{y}}{\bar{Y}}, C_{x}=\frac{S_{x}}{\bar{X}}, C_{z}=\frac{S_{z}}{\bar{Z}}, C_{y x}=\frac{\rho_{y x} C_{y}}{C_{x}}, C_{y z}=\frac{\rho_{y z} C_{y}}{C_{z}}, C_{x z}=\frac{\rho_{x z} C_{x}}{C_{z}}$, 


$$
\begin{aligned}
& \rho_{y x}=\frac{S_{y x}}{S_{x} S_{y}}, \rho_{y z}=\frac{S_{y z}}{S_{y} S_{z}}, \rho_{z x}=\frac{S_{z x}}{S_{z} S_{x}}, S_{y}^{2}=\frac{1}{N-1} \sum_{i=1}^{N}\left(y_{i}-\bar{Y}\right)^{2}, S_{x}^{2}=\frac{1}{N-1} \sum_{i=1}^{N}\left(x_{i}-\bar{X}\right)^{2}, \\
& S_{z}^{2}=\frac{1}{N-1} \sum_{i=1}^{N}\left(z_{i}-\bar{Z}\right)^{2}, S_{y x}=\frac{1}{N-1} \sum_{i=1}^{N}\left(y_{i}-\bar{Y}\right)\left(x_{i}-\bar{X}\right), \\
& S_{y z}=\frac{1}{N-1} \sum_{i=1}^{N}\left(y_{i}-\bar{Y}\right)\left(z_{i}-\bar{Z}\right) \text { and } S_{x z}=\frac{1}{N-1} \sum_{i=1}^{N}\left(x_{i}-\bar{X}\right)\left(z_{i}-\bar{Z}\right) .
\end{aligned}
$$

Expanding the right hand side ofequation (1) in terms of e's, multiplying out and neglecting the terms of e's of power greater than two,

$$
\left(t_{1}-\bar{Y}\right)=\bar{Y}=\left\{e_{0}+\frac{\lambda}{2}\left(e_{1}^{\prime}-e_{1}-e_{2}+e_{0} e_{1}^{\prime}-e_{0} e_{1}-e_{0} e_{2}\right)+\frac{1}{8}\left(\lambda^{2}-2 \lambda\right) e_{1}^{\prime 2}+\frac{1}{8}\left(\lambda^{2}+2 \lambda\right)\left(e_{1}^{2}+e_{2}^{2}\right)+\frac{\lambda^{2}}{4}\left(e_{1} e_{2}-e_{1} e_{1}^{\prime}-e_{1}^{\prime} e_{2}\right)\right\}
$$

where $\lambda=a \bar{X} /(2 b+a \bar{X})$.

Taking expectations in equation (3) and using the results of the equation (2), the Bias of the estimator $t_{1}$ to the first order approximation as

$$
B\left(t_{1}\right)_{I}=\bar{Y}\left\{\frac{1}{8}\left(\lambda^{2}+2 \lambda\right)\left(\frac{1-f^{*}}{n} C_{x}^{2}+\frac{1-f_{1}}{n_{1}} C_{z}^{2}\right)+\frac{\lambda}{2}\left(\frac{1-f^{*}}{n} C_{y x} C_{x}^{2}-\frac{1-f_{1}}{n_{1}} C_{y z} C_{z}^{2}\right)\right\}
$$

where, $f^{*}=n / n_{1}$.

From equation (3),

$$
\left(t_{1}-\bar{Y}\right) \cong \bar{Y}\left\{e_{0}+\frac{\lambda}{2}\left(e_{1}^{\prime}-e_{1}-e_{2}\right)\right\}
$$

Squaring both the sides of equation (4), taking expectations of its terms and using the results of equation (2), the MSE of $t_{1}$ to the first order approximation as

$$
M\left(t_{1}\right)_{I}=\bar{Y}^{2}\left\{\frac{1-f}{n} C_{y}^{2}+\frac{\lambda^{2}}{4}\left(\frac{1-f^{*}}{n} C_{x}^{2}+\frac{1-f_{1}}{n_{1}} C_{z}^{2}\right)-\lambda\left(\frac{1-f^{*}}{n} C_{y x} C_{x}^{2}+\frac{1-f_{1}}{n_{1}} C_{y z} C_{z}^{2}\right)\right\}
$$

Optimization of equation (5) with respect to $\lambda$ yields its optimum value as

$$
\lambda_{\text {opt. }}=\frac{2 A}{B}
$$

where, $A=\frac{1-f^{*}}{n} C_{y x} C_{x}^{2}+\frac{1-f_{1}}{n_{1}} C_{y z} C_{z}^{2}$ and $B=\frac{1-f^{*}}{n} C_{x}^{2}+\frac{1-f_{1}}{n_{1}} C_{z}^{2}$.

Substituting the value of $\lambda$ in equation (5), the optimum MSE of $t_{1}$ as

$$
\operatorname{opt} . M\left(t_{1}\right)_{I}=\bar{Y}^{2}\left(\frac{1-f}{n} C_{y}^{2}-\frac{A^{2}}{B}\right)
$$

\section{Efficiency Comparisons of $t_{1}$}

(i) with sample mean per unit estimator $\bar{y}$

The MSE of sample mean per unit estimator is

$$
M(\bar{y})=\bar{Y}^{2} \frac{1-f}{n} C_{y}^{2}
$$

From equations (7) and (8), it is found that the proposed estimator is more efficient than $\bar{y}$, since 
$M(\bar{y})-_{\text {opt. }} M\left(t_{1}\right)_{I}=\bar{Y}^{2} \frac{A^{2}}{B}>0$.

(ii) with chain ratio estimator in double sampling

The MSE of chain ratio estimator in double sampling is

$$
M\left(\hat{\bar{Y}}_{R}^{d c}\right)=\bar{Y}^{2}\left\{\frac{1-f}{n} C_{y}^{2}+\frac{1-f^{*}}{n} C_{x}^{2}\left(1-2 C_{y x}\right)+\frac{1-f_{1}}{n_{1}} C_{z}^{2}\left(1-2 C_{y z}\right)\right\}
$$

From equations (9) and (7),

$$
M\left(\hat{\bar{Y}}_{R}^{d c}\right)_{I}-_{o p t .} M\left(t_{1}\right)_{I}=\bar{Y}^{2}\left\{\frac{1-f^{*}}{n} C_{x}^{2}\left(1-2 C_{y x}\right)+\frac{1-f_{1}}{n_{1}} C_{z}^{2}\left(1-2 C_{y z}\right)+\frac{A^{2}}{B}\right\}
$$

Therefore, the proposed estimator is better than chain ratio type estimator if $1-2 C_{y x}>0$ and $1-2 C_{y z}>0$.

(iii) with exponential chain ratio estimator in double sampling

The MSE of exponential chain ratio estimator in double sampling is

$$
M\left(\hat{\bar{Y}}_{\mathrm{Re}}^{d c}\right)_{I}=\bar{Y}^{2}\left\{\frac{1-f}{n} C_{y}^{2}+\frac{1}{4}\left(\frac{1-f^{*}}{n} C_{x}^{2}+\frac{1-f_{1}}{n_{1}} C_{z}^{2}\right)-\left(\frac{1-f^{*}}{n} C_{y x} C_{x}^{2}+\frac{1-f_{1}}{n_{1}} C_{y z} C_{z}^{2}\right)\right\}
$$

From equations (10) and (7),

$$
M\left(\hat{\bar{Y}}_{\mathrm{Re}}^{d c}\right)_{I}-_{\text {opt. }} M\left(t_{1}\right)_{I}=\bar{Y}^{2} \frac{(A-B / 2)^{2}}{B}>0
$$

Therefore, the proposed estimator is more efficient than chain ratio estimator in double sampling.

\section{Bias and MSE of Case II}

In case II, let us write,

$$
\left.\begin{array}{l}
E\left(e_{0}\right)=E\left(e_{1}\right)=E\left(e_{1}^{\prime}\right)=E\left(e_{2}\right)=0, E\left(e_{0}^{2}\right)=\frac{1-f}{n} C_{y}^{2}, E\left(e_{1}^{2}\right)=\frac{1-f}{n} C_{x}^{2}, \\
E\left(e_{1}^{\prime 2}\right)=\frac{1-f_{1}}{n_{1}} C_{x}^{2}, E\left(e_{2}^{2}\right)=\frac{1-f_{1}}{n_{1}} C_{z}^{2}, E\left(e_{0} e_{1}\right)=\frac{1-f}{n} C_{y x} C_{x}^{2}, E\left(e_{1}^{\prime} e_{2}\right)=\frac{1-f_{1}}{n_{1}} C_{x z} C_{z}^{2} \\
E\left(e_{0} e_{1}^{\prime}\right)=E\left(e_{0} e_{2}\right)=E\left(e_{1} e_{1}^{\prime}\right)=E\left(e_{1} e_{2}\right)=0
\end{array}\right\}
$$

Taking expectations in equation (3) and using the results of the equation (11), the bias of the estimators $t_{1}$ to the first order approximation as

$$
B\left(t_{1}\right)_{I I}=\bar{Y}\left\{\frac{1}{8}\left(\lambda^{2}-2 \lambda\right) \frac{1-f_{1}}{n_{1}} C_{x}^{2}+\frac{1}{8}\left(\lambda^{2}+2 \lambda\right)\left(\frac{1-f}{n} C_{x}^{2}+\frac{1-f_{1}}{n_{1}} C_{z}^{2}\right)-\frac{\lambda}{2} \frac{1-f}{n} C_{y x} C_{x}^{2}-\frac{\lambda^{2}}{4} \frac{1-f_{1}}{n_{1}} C_{x z} C_{z}^{2}\right\}
$$

Squaring both the sides of equation (4), taking expectations of its terms and using the results of equation (11), the MSE of $t_{1}$ as

$$
M\left(t_{1}\right)_{I I}=\bar{Y}^{2}\left\{\frac{1-f}{n} C_{y}^{2}+\frac{\lambda^{2}}{4}\left(f^{* *} C_{x}^{2}+\frac{1-f_{1}}{n_{1}} C_{z}^{2}\right)-\lambda \frac{1-f}{n} C_{y x} C_{x}^{2}-\frac{\lambda^{2}}{2} \frac{1-f_{1}}{n_{1}} C_{x z} C_{z}^{2}\right\}
$$

where, $f^{* *}=\frac{1-f}{n}+\frac{1-f_{1}}{n_{1}}$.

Optimization of equation (12) with respect to $\lambda$ yields its optimum value as 


$$
\lambda_{\text {opt. }}=2 \frac{C}{D}
$$

where $C=\frac{1-f}{n} C_{y x} C_{x}^{2}$ and $D=f^{* *} C_{x}^{2}+\left(1-2 C_{x z}\right) \frac{1-f_{1}}{n_{1}} C_{z}^{2}$.

Substituting the value of equation (13) in equation (12), the optimum $\operatorname{MSE} M\left(t_{1}\right)_{I I}$ as

$$
\operatorname{opt.M}\left(t_{1}\right)_{I I}=\bar{Y}^{2}\left(\frac{1-f}{n} C_{y}^{2}-\frac{C^{2}}{D}\right)
$$

Efficiency Comparisons of $t_{1}$

(i) With sample mean per unit estimator $\bar{y}$

From equations (8) and (14), $M(\bar{y})-_{\text {opt. }} M\left(t_{1}\right)_{I I}=\bar{Y}^{2} \frac{C^{2}}{D}$.

Therefore, the proposed estimator is better than sample mean per unit estimator if $1-2 C_{x z}>0$.

(ii) With chain ratio estimator in double sampling

The MSE of chain ratio estimator in double sampling is

$$
M\left(\hat{\bar{Y}}_{R}^{d c}\right)_{I I}=\bar{Y}^{2}\left\{\frac{1-f}{n} C_{y}^{2}+\frac{1-f}{n} C_{x}^{2}\left(1-2 C_{y x}\right)+\frac{1-f_{1}}{n_{1}} C_{x}^{2}+\frac{1-f_{1}}{n_{1}} C_{z}^{2}\left(1-2 C_{x z}\right)\right\}
$$

From equations (15) and (14), we have

$$
M\left(\hat{\bar{Y}}_{R}^{d c}\right)_{I I}-{ }_{o p t .} M\left(t_{1}\right)_{I I}=\bar{Y}^{2}\left[\frac{1-f}{n} C_{x}^{2}\left(1-2 C_{y x}\right)+\frac{1-f_{1}}{n_{1}}\left\{C_{x}^{2}+C_{z}^{2}\left(1-2 C_{x z}\right)\right\}+\frac{C^{2}}{D}\right]
$$

Therefore, the proposed estimator is better than the chain ratio estimator if $1-2 C_{y x}>0$ and $1-2 C_{x z}>0$.

(iii) With exponential chain ratio estimator in double sampling

The MSE of exponential chain ratio estimator in double sampling is

$$
M\left(\hat{\bar{Y}}_{\mathrm{Re}}^{d c}\right)_{I I}=\bar{Y}^{2}\left\{\frac{1-f}{n} C_{y}^{2}+\frac{1}{4}\left(f^{* *} C_{x}^{2}+\frac{1-f_{1}}{n_{1}} C_{z}^{2}\right)-\frac{1-f}{n} C_{y x} C_{x}^{2}-\frac{1}{2} \frac{1-f_{1}}{n_{1}} C_{x z} C_{z}^{2}\right\}
$$

From equations (14) and (16), we have

$$
M\left(\hat{\bar{Y}}_{\mathrm{Re}}^{d c}\right)_{I I}-_{o p t .} M\left(t_{1}\right)_{I I}=\bar{Y}^{2}\left\{\frac{1}{4}\left(f^{* *} C_{x}^{2}+\frac{1-f_{1}}{n_{1}} C_{z}^{2}\right)-\left(\frac{1-f}{n} C_{y x} C_{x}^{2}+\frac{1}{2} \frac{1-f_{1}}{n_{1}} C_{x z} C_{z}^{2}\right)+\frac{C^{2}}{D}\right\}
$$

Therefore, the proposed estimator is more efficient than exponential chain ratio estimator in double sampling if $C_{y x}<0$ and $C_{x z}<0$.

\section{Empirical Study}

To examine the merits of the proposed estimator, we have considered five natural population data sets. The sources of populations, nature of the variates $y, x$ and $z$; and the values of the various parameters are given as follows.

Population I- Source: Murthy [10]

$Y$ : Area under wheat in 1964

$X$ : Area under wheat in 1963

$Z$ : Cultivated area in 1961

$$
\begin{aligned}
& N=34, n=7, n_{1}=10, \bar{Y}=199.44 \text { acre }, \bar{X}=208.89 \text { acre, } \bar{Z}=747.59 \text { acre }, \\
& \rho_{y x}=0.9801, \rho_{y z}=0.9043, \rho_{x z}=0.9097, C_{y}^{2}=0.5673, C_{x}^{2}=0.5191, C_{z}^{2}=0.3527 .
\end{aligned}
$$


Population II- Source: Cochran [5]

$Y$ : Number of 'placebo' children

$X:$ Number of paralytic polio cases in the placebo group

$Z$ : Number of paralytic polio cases in the 'not inoculated' group

$$
\begin{aligned}
& N=34, \quad n=10, n_{1}=15, \bar{Y}=4.92, \bar{X}=2.59, \bar{Z}=2.91, \rho_{y x}=0.7326, \rho_{y z}=0.6430, \\
& \rho_{x z}=0.6837, C_{y}^{2}=1.0248, \quad C_{x}^{2}=1.5175, \quad C_{z}^{2}=1.1492 .
\end{aligned}
$$

Population III- Source: Sukhatme and Chand [16]

$Y$ : Apple trees of bearing age in 1964

$X$ : Bushels of apples harvested in 1964

$Z$ : Bushels of apples harvested in 1959

$$
\begin{aligned}
& N=200, \quad n=20, \quad n_{1}=30, \bar{Y}=0.103182 \times 10^{4}, \bar{X}=0.293458 \times 10^{4}, \bar{Z}=0.365149 \times 10^{4}, \\
& \rho_{y x}=0.93, \quad \rho_{y z}=0.77, \quad \rho_{x z}=0.84, \quad C_{y}^{2}=2.55280, \quad C_{x}^{2}=4.02504, C_{z}^{2}=2.09379 .
\end{aligned}
$$

Population IV-Source: Srivastava et al.[18]

$Y$ : The measurement of weight of children

$X:$ Mid arm circumference of children

$Z$ : Skull circumference of children.

$$
\begin{aligned}
& N=82, \quad n=25, \quad n_{1}=43, \bar{Y}=5.60 \mathrm{~kg}, \bar{X}=11.90 \mathrm{~cm}, \bar{Z}=39.80 \mathrm{~cm}, \rho_{y x}=0.09 \\
& \rho_{y z}=0.12, \quad \rho_{x z}=0.86, C_{y}^{2}=0.0107, \quad C_{x}^{2}=0.0052, C_{z}^{2}=0.0008
\end{aligned}
$$

Population $V$ - Source: Srivastava et al.[15]

$Y$ : The measurement of weight of children

$X$ : Mid arm circumference of children

$Z$ : Skull circumference of children.

$$
\begin{aligned}
& N=55, \quad n=18, \quad n_{1}=30, \quad \bar{Y}=17.08 \mathrm{~kg}, \bar{X}=16.92 \mathrm{~cm}, \bar{Z}=50.44 \mathrm{~cm}, \rho_{y x}=0.54, \\
& \rho_{y z}=0.51, \quad \rho_{x z}=-0.08, \quad C_{y}^{2}=0.0161, \quad C_{x}^{2}=0.0049, \quad C_{z}^{2}=0.0007 .
\end{aligned}
$$

To observe the relative performance of different estimators of $\bar{Y}$, we have computed the percentage relative efficiencies of the proposed estimator $\left(t_{1}\right)$, exponential chain ratio type estimator $\left(\hat{\bar{Y}}_{R e}^{d c}\right)$, chain ratio estimator $\left(\hat{\bar{Y}}_{R}^{d c}\right)$ in double samplingand sample mean per unit estimator $\bar{y}$ with respect to usual unbiased estimator $\bar{y}$. The findings are presented in Table 1 and 2 .

Table 1. Percentage relative efficiencies of different estimators w. r.t. $\bar{y}$ for Case I.

\begin{tabular}{lllll}
\hline Estimators $\rightarrow$ & $\bar{y}$ & $\hat{\bar{Y}}_{R}^{d c}$ & $\hat{\bar{Y}}_{R e}^{d c}$ & $t_{1}$ \\
\hline Population I & 100.00 & 730.81 & 259.55 & 763.30 \\
Population II & 100.00 & 136.91 & 184.36 & 189.27 \\
Population III & 100.00 & 279.93 & 247.82 & 322.94 \\
Population IV & 100.00 & 81.92 & 97.11 & 100.81 \\
Population V & 100.00 & 131.91 & 120.57 & 132.32 \\
\hline
\end{tabular}

Table 2. Percentage relative efficiencies of different estimators w. r. t. $\bar{y}$ for Case II.

\begin{tabular}{lllll}
\hline Estimators $\rightarrow$ & $\bar{y}$ & $\hat{\bar{Y}}_{R}^{d c}$ & $\hat{\bar{Y}}_{R e}^{d c}$ & $t_{1}$ \\
\hline Population I & 100.00 & 702.77 & 236.11 & 735.13 \\
Population II & 100.00 & 87.63 & 141.68 & 170.37 \\
Population III & 100.00 & 182.67 & 220.59 & 362.61 \\
Population IV & 100.00 & 68.82 & 91.06 & 100.68 \\
Population V & 100.00 & 116.68 & 122.79 & 124.38 \\
\hline
\end{tabular}




\section{Conclusions}

From Table 1 and Table 2, it is seen that the percentage relative efficiencies of proposed class of estimator is higher than the other estimators in all the population data sets considered in this paper. So it is evident that the proposed class of exponential chain ratio type estimators $t_{1}$ is more efficient than the sample mean per unit estimator $\bar{y}$, chain ratio type estimator $\left(\hat{\bar{Y}}_{R}^{d c}\right)$ proposed by Chand [4] and exponential chain ratio type estimator $\left(\hat{\bar{Y}}_{R e}^{d c}\right)$ proposed by Singh \& Choudhury [12] for both the cases under double sampling. Thus, the uses of the proposed estimators are preferable over other competitive estimators.

\section{References}

[1] Al-Jararha, J. \& Ahmed, M. S. (2002). The class of chain estimators for a finite population variance using double sampling. Information and Management Sciences, 13(2), 1318.

[2] Amin, M. \& Hanif, M. (2012). Some exponential estimators in survey sampling. Pak. J. Statist. 28(3), 67-374.

[3] Bahl, S. \&Tuteja, R. K. (1991). Ratio and Product type exponential estimator. Information and Optimization Sciences, $12,159-163$.

[4] Chand, L. (1975). Some ratio type estimator based on two or more auxiliary variables. Unpublished Ph. D. dissertation, Lowa State University, Ames, Lowa.

[5] Cochran, W. G. (1940). The estimation of the yields of the cereal experiments by sampling for the ratio of grain to total produce. The Journal of Agricultural Science, 30(2), 262-275.

[6] Cochran, W. G. (1977). Sampling techniques. New-York: John Wiley and Sons.

[7] J. Neyman. (1938). Contribution to the theory of sampling human populations. Journal of the American Statistical Association, (33), 101-116.

[8] Kalita, et al. (2013). Improved exponential chain ratio and Product type Estimators for finite Population mean in double sampling. International Journal of Statistical Sciences, 13, 2127.

[9] Kiregyera, B. (1980). A chain ratio-type estimator in finite population in double sampling using two auxiliary variables. Metrika, 27, 217-223.

[10] Murthy, M. N. (1967). Sampling theory and Methods. Calcutta, India: Statistical Publishing Soc.

[11] Pradhan, B. K. (2005). A Chain Regression Estimator in Two Phase Sampling Using Multiauxiliary Information. Bulletin of the Malaysian Mathematical Sciences Society (2), 28(1), 8186.

[12] Singh, B.K. \& Choudhury. S, (2012). Exponential chain ratio and product type estimators for finite population mean under double sampling scheme, Global Journal of Science Frontier Research, 12(6), 13-24.

[13] Singh, H. P. \& Vishwakarma, G. K. (2007). Modified exponential ratio and product estimators for finite population mean in double sampling. Austrian Journal of Statistics, 36(3), 217-225.

[14] Srivastava, S. K. \& Jhajj, H. S. (1980). A class of estimators using auxiliary information for estimating finite population variance. Sankhyā C, 42(1-2), 87-96.

[15] Srivastava, et.al (1989). Chain ratio type estimator for ratio of two population means using auxiliary characters. Communications in Statistics -Theory and Methods, 18(10), 3917-3926.

[16] Sukhatme, B. V. \& Chand, L. (1977). Multivariate ratio-type estimators, Proceedings of American Statistical Association, Social Statistics Section, 927-931.

[17] Sukhatme, B. V. (1962). Some ratio-type estimators in twophase sampling. J. Amer. Statist. Assoc., 57, 628-632.

[18] Swain, A. K. P. C. (1970). A note on the use of multiple auxiliary variables in sample surveys. Trabajos de Estadist., Inves. Oper., 21(3), 135-14. 